\title{
Strong Convergence Theorems for Equilibrium Problems and Fixed Point Problems in Hilbert Spaces
}

\author{
Jian-Wen Peng' and Yan Wang ${ }^{2}$ \\ ${ }^{1}$ College of Mathematics and Computer Science, Chongqing Normal University, Chongqing 400047, China \\ 2 Division of Mathematics, Yibin No. 3 Middle School, Yibin, Sichuan 644000, China
}

Correspondence should be addressed to Jian-Wen Peng, jwpeng6@yahoo.com.cn

Received 21 August 2009; Revised 1 December 2009; Accepted 13 January 2010

Academic Editor: Petru Jebelean

Copyright (c) 2010 J.-W. Peng and Y. Wang. This is an open access article distributed under the Creative Commons Attribution License, which permits unrestricted use, distribution, and reproduction in any medium, provided the original work is properly cited.

We introduce an Ishikawa iterative scheme by the viscosity approximate method for finding a common element of the set of solutions of an equilibrium problem and the set of fixed points of a nonexpansive mapping in Hilbert space. Then, we prove some strong convergence theorems which extend and generalize S. Takahashi and W. Takahashi's results (2007).

\section{Introduction}

Let $H$ be a real Hilbert space and let $C$ be a nonempty closed convex subset of $H$. Let $F$ be a bifunction from $C \times C$ to $R$, where $R$ is the set of real numbers. The equilibrium problem for $F: C \times C \rightarrow R$ is to find $x \in C$ such that

$$
F(x, y) \geq 0, \quad \forall y \in C
$$

The set of solutions of (1.1) is denoted by $\operatorname{EP}(F)$. Given a mapping $T: C \rightarrow H$, let $F(x, y)=\langle T x, y-x\rangle$ for all $x, y \in C$. Then, $z \in \operatorname{EP}(F)$ if and only if $\langle T z, y-z\rangle \geq 0$ for all $y \in C$. Numerous problems in physics, optimization, and economics reduce to find a solution of (1.1); for more details, see [1,2].

Recall that a self-mapping $S$ of a closed convex subset $C$ of $H$ is nonexpansive [3] if there holds that

$$
\|S x-S y\| \leq\|x-y\|, \quad \forall x, y \in C .
$$


We denote the set of fixed points of $S$ by $F(S)$. There are some methods for approximation of fixed points of a nonexpansive mapping. In 2000, Moudafi [4] introduced the viscosity approximation method for nonexpansive mappings (see [5] for further developments in both Hilbert and Banach spaces). Some methods have been proposed to solve the equilibrium problem; see, for instance, [1, 2, 6, 7]. Recently, Combettes and Hirstoaga [6] introduced an iterative scheme of finding the best approximation to the initial data when $\operatorname{EP}(F)$ is nonempty and proved a strong convergence theorem. S. Takahashi and W. Takahashi [7] introduced a Mann iterative scheme by the viscosity approximation method for finding a common element of the set of solution (1.1) and the set of fixed points of a nonexpansive mapping in a Hilbert space and proved a strong convergence theorem.

On the other hand, Ishikawa [8] introduced the following iterative process defined recursively by

$$
\begin{gathered}
x_{n+1}=\alpha_{n} x_{n}+\left(1-\alpha_{n}\right) S y_{n}, \\
y_{n}=\beta_{n} x_{n}+\left(1-\beta_{n}\right) S x_{n}, \quad \forall n \in N,
\end{gathered}
$$

where the initial guess $x_{0}$ is taking in $C$ arbitrarily, $\left\{\alpha_{n}\right\}$ and $\left\{\beta_{n}\right\}$ are sequences in the interval $[0,1]$.

In this paper, motivated by the ideas in [4-8], we introduce an Ishikawa iterative scheme by the viscosity approximation method for finding a common element of the set of solution (1.1) and the set of fixed points of a nonexpansive mapping in a Hilbert space.

Starting with an arbitrary $x_{1} \in H$, define sequences $\left\{x_{n}\right\},\left\{y_{n}\right\}$, and $\left\{u_{n}\right\}$ by

$$
\begin{gathered}
F\left(u_{n}, y\right)+\frac{1}{r_{n}}\left\langle y-u_{n}, u_{n}-x_{n}\right\rangle \geq 0, \quad \forall y \in C, \\
x_{n+1}=\alpha_{n} f\left(x_{n}\right)+\left(1-\alpha_{n}\right) S y_{n}, \\
y_{n}=\beta_{n} x_{n}+\left(1-\beta_{n}\right) S u_{n}, \quad \forall n \in N,
\end{gathered}
$$

where $\left\{\alpha_{n}\right\},\left\{\beta_{n}\right\} \subset[0,1]$ and $\left\{r_{n}\right\} \subset(0, \infty)$.

We will prove in Section 3 that if the sequences $\left\{\alpha_{n}\right\},\left\{\beta_{n}\right\}$, and $\left\{r_{n}\right\}$ of parameters satisfy appropriate conditions, then the sequences $\left\{x_{n}\right\},\left\{y_{n}\right\}$, and $\left\{u_{n}\right\}$ generated by (1.4) converge strongly to $z \in F(S) \cap \operatorname{EP}(F)$. The results in this paper extend and generalize $\mathrm{S}$. Takahashi and W. Takahashi's results [7].

\section{Preliminaries}

Let $H$ be a real Hilbert space with inner product $\langle\cdot, \cdot\rangle$, and norm $\|\cdot\|$ and let $C$ be a nonempty closed convex subset of $H . x_{n} \rightarrow x$ implies that $\left\{x_{n}\right\}$ converges strongly to $x$ and $x_{n} \rightarrow x$ means that $\left\{x_{n}\right\}$ converges weakly to $x$. In a real Hilbert space $H$, we have

$$
\|\lambda x+(1-\lambda) y\|^{2}=\lambda\|x\|^{2}+(1-\lambda)\|y\|^{2}-\lambda(1-\lambda)\|x-y\|^{2}
$$

for all $x, y \in H$ and $\lambda \in R$; see [9]. 
For any $x \in H$, there exists a unique nearest point in $C$, denoted by $P_{C}(x)$, such that $\left\|x-P_{C}(x)\right\| \leq\|x-y\|$ for all $y \in C$. Such a $P_{C}$ is called the metric projection of $H$ onto $C$. It is also known that $y=P_{C}(x)$ is equivalent to $\langle x-y, y-z\rangle \geq 0$ for all $z \in C$.

For solving the equilibrium problem, let us assume that the bifunction $F$ satisfies the following conditions:

(A1) $F(x, x)=0$ for all $x \in C$;

(A2) $F$ is monotone, that is, $F(x, y)+F(y, x) \leq 0$ for any $x, y \in C$;

(A3) for each $x, y, z \in C$,

$$
\lim _{t \downarrow 0} F(t z+(1-t) x, y) \leq F(x, y)
$$

(A4) for each $x \in C, y \mapsto F(x, y)$ is convex and lower semicontinuous.

We recall some lemmas needed later.

Lemma 2.1 (see [2]). Let $C$ be a nonempty closed convex subset of $H$ and let $F$ be a bifunction from $C \times C$ to $R$ satisfying (A1)-(A4). Let $r>0$ and $x \in H$. Then, there exists $z \in C$ such that

$$
F(z, y)+\frac{1}{r}\langle y-z, z-x\rangle \geq 0, \quad \forall y \in C
$$

Lemma 2.2 (see [5]). Let $C$ be a nonempty closed convex subset of $H$, and let $F$ be a bifunction from $C \times C$ to $R$ satisfying (A1)-(A4). For $r>0$ and $x \in H$, define a mapping $T_{r}: H \rightarrow C$ as follows:

$$
T_{r}(x)=\left\{z \in C: F(z, y)+\frac{1}{r}\langle y-z, z-x\rangle \geq 0, \forall y \in C\right\}
$$

for all $x \in H$. Then, the following statements hold:

(1) $T_{r}$ is single-valued;

(2) $T_{r}$ is firmly nonexpansive, that is, for any $x, y \in H$,

$$
\left\|T_{r}(x)-T_{r}(y)\right\|^{2} \leq\left\langle T_{r}(x)-T_{r}(y), x-y\right\rangle ;
$$

(3) $F\left(T_{r}\right)=\mathrm{EP}(F)$;

(4) $\mathrm{EP}(F)$ is closed and convex.

Lemma 2.3 (see [10]). Let $\left\{a_{n}\right\}$ be a sequence of nonnegative real numbers such that

$$
a_{n+1} \leq\left(1-c_{n}\right) a_{n}+b_{n}, \quad \forall n \in N,
$$


where $\left\{b_{n}\right\}$ is a sequence of real numbers and $\left\{c_{n}\right\}$ is a sequence in $(0,1)$ such that

(i) $\sum_{n=1}^{\infty} c_{n}=\infty$,

(ii) $\lim \sup _{n \rightarrow \infty}\left(b_{n} / c_{n}\right) \leq 0$ or $\sum_{n=1}^{\infty}\left|b_{n}\right|<\infty$.

Then, $\lim _{n \rightarrow \infty} a_{n}=0$.

\section{Strong Convergence Theorem}

In this section, we show a strong convergence theorem which solves the problem of finding a common element of the set of solutions of an equilibrium problem and the set of fixed points of a nonexpansive mapping in a Hilbert space.

Theorem 3.1. Let $C$ be a nonempty closed convex subset of $H$. Let $F$ be a bifunction from $C \times C$ to $R$ satisfying (A1)-(A4) and let $S$ be a nonexpansive mapping of $C$ into $H$ such that $F(S) \cap \operatorname{EP}(F) \neq \emptyset$. Let $f$ be a contraction of $H$ into itself and let $\left\{x_{n}\right\},\left\{u_{n}\right\}$, and $\left\{y_{n}\right\}$ be sequences generated by $x_{1} \in H$ and (1.4). If $\left\{\alpha_{n}\right\},\left\{\beta_{n}\right\} \subset[0,1]$ and $\left\{r_{n}\right\} \subset(0, \infty)$ satisfy the following conditions:

$$
\begin{gathered}
\lim _{n \rightarrow \infty} \alpha_{n}=0, \quad \sum_{n=1}^{\infty} \alpha_{n}=\infty, \quad \sum_{n=1}^{\infty}\left|\alpha_{n+1}-\alpha_{n}\right|<\infty, \quad \sum_{n=1}^{\infty}\left|\beta_{n+1}-\beta_{n}\right|<\infty, \\
0<\liminf _{n \rightarrow \infty} \beta_{n} \leq \limsup _{n \rightarrow \infty} \beta_{n}<1, \quad \sum_{n=1}^{\infty}\left|r_{n+1}-r_{n}\right|<\infty,
\end{gathered}
$$

then, $\left\{x_{n}\right\},\left\{y_{n}\right\}$, and $\left\{u_{n}\right\}$ converge strongly to $z \in F(S) \cap \operatorname{EP}(F)$, where $z=P_{F(S) \cap \operatorname{EP}(F)} f(z)$.

Proof. Let $Q=P_{F(S) \cap E P(F)}$. Then $Q f$ is a contraction of $H$ into itself. In fact, there exists $a \in$ $[0,1)$ such that $\|f(x)-f(y)\| \leq a\|x-y\|$ for all $x, y \in H$. So, we have that

$$
\|Q f(x)-Q f(y)\| \leq\|f(x)-f(y)\| \leq a\|x-y\|
$$

for all $x, y \in H$. Since $H$ is complete, there exists a unique element $z \in H$ such that $z=Q f(z)$. Such a $z \in H$ is an element of $C$.

Let $v \in F(S) \cap E P(F)$. Then from $u_{n}=T_{r_{n}} x_{n}$, we have

$$
\left\|u_{n}-v\right\|=\left\|T_{r_{n}} x_{n}-T_{r_{n}} v\right\| \leq\left\|x_{n}-v\right\|
$$

for all $n \in N$. Put $M=\max \left\{\left\|x_{1}-v\right\|,(1 /(1-a))\|f(v)-v\|\right\}$. It is obvious that $\left\|x_{1}-v\right\| \leq M$. 
Suppose $\left\|x_{n}-v\right\| \leq M$. Then, we have

$$
\begin{aligned}
\left\|x_{n+1}-v\right\| & \leq \alpha_{n}\left\|f\left(x_{n}\right)-v\right\|+\left(1-\alpha_{n}\right)\left\|S y_{n}-v\right\| \\
& \leq \alpha_{n}\left\|f\left(x_{n}\right)-f(v)\right\|+\alpha_{n}\|f(v)-v\|+\left(1-\alpha_{n}\right)\left\|S y_{n}-v\right\| \\
& \leq a \alpha_{n}\left\|x_{n}-v\right\|+\alpha_{n}\|f(v)-v\|+\left(1-\alpha_{n}\right)\left\|y_{n}-v\right\| .
\end{aligned}
$$

On the other hand

$$
\begin{aligned}
\left\|y_{n}-v\right\| & \leq \beta_{n}\left\|x_{n}-v\right\|+\left(1-\beta_{n}\right)\left\|S u_{n}-v\right\| \\
& \leq \beta_{n}\left\|x_{n}-v\right\|+\left(1-\beta_{n}\right)\left\|u_{n}-v\right\| \\
& \leq \beta_{n}\left\|x_{n}-v\right\|+\left(1-\beta_{n}\right)\left\|x_{n}-v\right\| \\
& =\left\|x_{n}-v\right\| .
\end{aligned}
$$

Putting (3.5) into (3.4), we have

$$
\begin{aligned}
\left\|x_{n+1}-v\right\| & \leq a \alpha_{n}\left\|x_{n}-v\right\|+\alpha_{n}\|f(v)-v\|+\left(1-\alpha_{n}\right)\left\|x_{n}-v\right\| \\
& =\left[1-\alpha_{n}(1-a)\right]\left\|x_{n}-v\right\|+\alpha_{n}(1-a) \frac{\|f(v)-v\|}{1-a} \\
& \leq\left[1-\alpha_{n}(1-a)\right] M+\alpha_{n}(1-a) M=M .
\end{aligned}
$$

So, we have that $\left\|x_{n+1}-v\right\| \leq M$ for any $n \in N$. And hence $\left\{x_{n}\right\}$ is bounded. We also obtain that $\left\{u_{n}\right\},\left\{y_{n}\right\},\left\{S u_{n}\right\},\left\{S y_{n}\right\}$, and $\left\{f\left(x_{n}\right)\right\}$ are bounded. Next, we show that $\lim _{n \rightarrow \infty} \| x_{n+1}-$ $x_{n} \|=0$. In fact,

$$
\begin{aligned}
\left\|y_{n}-y_{n-1}\right\| & =\left\|\beta_{n} x_{n}+\left(1-\beta_{n}\right) S u_{n}-\left[\beta_{n-1} x_{n-1}+\left(1-\beta_{n-1}\right) S u_{n-1}\right]\right\| \\
& =\left\|\beta_{n}\left(x_{n}-x_{n-1}\right)+\left(\beta_{n}-\beta_{n-1}\right) x_{n-1}+\left(1-\beta_{n}\right)\left(S u_{n}-S u_{n-1}\right)+\left(\beta_{n-1}-\beta_{n}\right) S u_{n-1}\right\| \\
& \leq\left|\beta_{n}-\beta_{n-1}\right|\left\|x_{n-1}\right\|+\beta_{n}\left\|x_{n}-x_{n-1}\right\|+\left(1-\beta_{n}\right)\left\|u_{n}-u_{n-1}\right\|+\left|\beta_{n}-\beta_{n-1}\right|\left\|S u_{n-1}\right\|,
\end{aligned}
$$


and hence

$$
\begin{aligned}
\left\|x_{n+1}-x_{n}\right\|= & \left\|\alpha_{n} f\left(x_{n}\right)+\left(1-\alpha_{n}\right) S y_{n}-\alpha_{n-1} f\left(x_{n-1}\right)-\left(1-\alpha_{n-1}\right) S y_{n-1}\right\| \\
= & \| \alpha_{n} f\left(x_{n}\right)-\alpha_{n} f\left(x_{n-1}\right)+\alpha_{n} f\left(x_{n-1}\right)-\alpha_{n-1} f\left(x_{n-1}\right) \\
& +\left(1-\alpha_{n}\right) S y_{n}-\left(1-\alpha_{n}\right) S y_{n-1}+\left(1-\alpha_{n}\right) S y_{n-1}-\left(1-\alpha_{n-1}\right) S y_{n-1} \| \\
\leq & \alpha_{n} a\left\|x_{n}-x_{n-1}\right\|+\left|\alpha_{n}-\alpha_{n-1}\right|\left\|f\left(x_{n-1}\right)\right\|+\left(1-\alpha_{n}\right)\left\|y_{n}-y_{n-1}\right\| \\
& +\left|\alpha_{n}-\alpha_{n-1}\right|\left\|S y_{n-1}\right\| \\
\leq & \alpha_{n} a\left\|x_{n}-x_{n-1}\right\|+\left|\alpha_{n}-\alpha_{n-1}\right|\left\|f\left(x_{n-1}\right)\right\|+\left(1-\alpha_{n}\right) \\
& \times\left[\left|\beta_{n}-\beta_{n-1}\right|\left\|x_{n-1}\right\|+\beta_{n}\left\|x_{n}-x_{n-1}\right\|+\left(1-\beta_{n}\right)\left\|u_{n}-u_{n-1}\right\|+\left|\beta_{n}-\beta_{n-1}\right|\left\|S u_{n-1}\right\|\right] \\
& +\left|\alpha_{n}-\alpha_{n-1}\right|\left\|S y_{n-1}\right\| \\
= & {\left[\beta_{n}-\alpha_{n}\left(\beta_{n}-a\right)\right]\left\|x_{n}-x_{n-1}\right\|+\left|\alpha_{n}-\alpha_{n-1}\right|\left[\left\|f\left(x_{n-1}\right)\right\|+\left\|S y_{n-1}\right\|\right] } \\
& +\left(1-\alpha_{n}\right)\left|\beta_{n}-\beta_{n-1}\right|\left[\left\|x_{n-1}\right\|+\left\|S u_{n-1}\right\|\right]+\left(1-\alpha_{n}\right)\left(1-\beta_{n}\right)\left\|u_{n}-u_{n-1}\right\| \\
\leq & {\left[\beta_{n}-\alpha_{n}\left(\beta_{n}-a\right)\right]\left\|x_{n}-x_{n-1}\right\|+\left|\alpha_{n}-\alpha_{n-1}\right| K_{1}+\left(1-\alpha_{n}\right)\left|\beta_{n}-\beta_{n-1}\right| K_{2} } \\
& +\left(1-\alpha_{n}\right)\left(1-\beta_{n}\right)\left\|u_{n}-u_{n-1}\right\|,
\end{aligned}
$$

where $K_{1}=\sup \left\{\left\|f\left(x_{n}\right)\right\|+\left\|S y_{n}\right\|: n \in N\right\}$ and $K_{2}=\sup \left\{\left\|x_{n}\right\|+\left\|S u_{n}\right\|: n \in N\right\}$.

On the other hand, from $u_{n}=T_{r_{n}} x_{n}$ and $u_{n+1}=T_{r_{n+1}} x_{n+1}$, we have

$$
\begin{gathered}
F\left(u_{n}, y\right)+\frac{1}{r_{n}}\left\langle y-u_{n}, u_{n}-x_{n}\right\rangle \geq 0, \quad \forall y \in C, \\
F\left(u_{n+1}, y\right)+\frac{1}{r_{n+1}}\left\langle y-u_{n+1}, u_{n+1}-x_{n+1}\right\rangle \geq 0, \quad \forall y \in C .
\end{gathered}
$$

Putting $y=u_{n+1}$ in (3.9) and $y=u_{n}$ in (3.10), we have

$$
\begin{gathered}
F\left(u_{n}, u_{n+1}\right)+\frac{1}{r_{n}}\left\langle u_{n+1}-u_{n}, u_{n}-x_{n}\right\rangle \geq 0, \\
F\left(u_{n+1}, u_{n}\right)+\frac{1}{r_{n+1}}\left\langle u_{n}-u_{n+1}, u_{n+1}-x_{n+1}\right\rangle \geq 0 .
\end{gathered}
$$

So, from the monotonicity of $F$, we get

$$
\left\langle u_{n+1}-u_{n}, \frac{u_{n}-x_{n}}{r_{n}}-\frac{u_{n+1}-x_{n+1}}{r_{n+1}}\right\rangle \geq 0,
$$


and hence

$$
\left\langle u_{n+1}-u_{n}, u_{n}-u_{n+1}+u_{n+1}-x_{n}-\frac{r_{n}}{r_{n+1}}\left(u_{n+1}-x_{n+1}\right)\right\rangle \geq 0
$$

Without loss of generality, let us assume that there exists a real number $b$ such that $r_{n}>b>0$ for all $n \in N$. Then, we have

$$
\begin{aligned}
\left\|u_{n+1}-u_{n}\right\|^{2} & \leq\left\langle u_{n+1}-u_{n}, x_{n+1}-x_{n}+\left(1-\frac{r_{n}}{r_{n+1}}\right)\left(u_{n+1}-x_{n+1}\right)\right\rangle \\
& \leq\left\|u_{n+1}-u_{n}\right\|\left\{\left\|x_{n+1}-x_{n}\right\|+\left|1-\frac{r_{n}}{r_{n+1}}\right|\left\|u_{n+1}-x_{n+1}\right\|\right\},
\end{aligned}
$$

and hence

$$
\begin{aligned}
\left\|u_{n+1}-u_{n}\right\| & \leq\left\|x_{n+1}-x_{n}\right\|+\frac{1}{r_{n+1}}\left|r_{n+1}-r_{n}\right|\left\|u_{n+1}-x_{n+1}\right\| \\
& \leq\left\|x_{n+1}-x_{n}\right\|+\frac{1}{b}\left|r_{n+1}-r_{n}\right| L
\end{aligned}
$$

where $L=\sup \left\{\left\|u_{n}-x_{n}\right\|: n \in N\right\}$. So from (3.8), we have

$$
\begin{aligned}
\left\|x_{n+1}-x_{n}\right\| \leq & {\left[\beta_{n}-\alpha_{n}\left(\beta_{n}-a\right)\right]\left\|x_{n}-x_{n-1}\right\|+\left|\alpha_{n}-\alpha_{n-1}\right| K_{1} } \\
& +\left(1-\alpha_{n}\right)\left|\beta_{n}-\beta_{n-1}\right| K_{2}+\left(1-\alpha_{n}\right)\left(1-\beta_{n}\right)\left[\left\|x_{n}-x_{n-1}\right\|+\frac{1}{b}\left|r_{n}-r_{n-1}\right| L\right] \\
= & \left(1-\alpha_{n}(1-a)\right)\left\|x_{n}-x_{n-1}\right\|+\left|\alpha_{n}-\alpha_{n-1}\right| K_{1} \\
& +\left(1-\alpha_{n}\right)\left|\beta_{n}-\beta_{n-1}\right| K_{2}+\left(1-\alpha_{n}\right)\left(1-\beta_{n}\right) \frac{1}{b}\left|r_{n}-r_{n-1}\right| L .
\end{aligned}
$$

Using Lemma 2.1 in [10], we obtain

$$
\lim _{n \rightarrow \infty}\left\|x_{n+1}-x_{n}\right\|=0
$$

From (3.15) and $\left|r_{n+1}-r_{n}\right| \rightarrow 0$, we have

$$
\lim _{n \rightarrow \infty}\left\|u_{n+1}-u_{n}\right\|=0
$$

It follows from (3.7) that

$$
\lim _{n \rightarrow \infty}\left\|y_{n+1}-y_{n}\right\|=0
$$


Since $x_{n}=\alpha_{n-1} f\left(x_{n-1}\right)+\left(1-\alpha_{n-1}\right) S y_{n-1}$, we have

$$
\begin{aligned}
\left\|x_{n}-S y_{n}\right\| & \leq\left\|x_{n}-S y_{n-1}\right\|+\left\|S y_{n-1}-S y_{n}\right\| \\
& \leq \alpha_{n-1}\left\|f\left(x_{n-1}\right)-S y_{n-1}\right\|+\left\|y_{n-1}-y_{n}\right\| .
\end{aligned}
$$

From $\alpha_{n} \rightarrow 0$, we have $\left\|x_{n}-S y_{n}\right\| \rightarrow 0$. For $v \in F(S) \cap E P(F)$, we have

$$
\begin{aligned}
\left\|u_{n}-v\right\|^{2} & =\left\|T_{r_{n}} x_{n}-T_{\mathrm{r}_{n}} v\right\|^{2} \\
& \leq\left\langle T_{r_{n}} x_{n}-T_{r_{n}} v, x_{n}-v\right\rangle \\
& =\left\langle u_{n}-v, x_{n}-v\right\rangle \\
& =\frac{1}{2}\left(\left\|u_{n}-v\right\|^{2}+\left\|x_{n}-v\right\|^{2}-\left\|x_{n}-u_{n}\right\|^{2}\right),
\end{aligned}
$$

and hence

$$
\left\|u_{n}-v\right\|^{2} \leq\left\|x_{n}-v\right\|^{2}-\left\|x_{n}-u_{n}\right\|^{2} .
$$

Therefore, from the convexity of $\|\cdot\|^{2}$, we have

$$
\begin{aligned}
\left\|y_{n}-v\right\|^{2} & \leq \beta_{n}\left\|x_{n}-v\right\|^{2}+\left(1-\beta_{n}\right)\left\|S u_{n}-v\right\|^{2} \\
& \leq \beta_{n}\left\|x_{n}-v\right\|^{2}+\left(1-\beta_{n}\right)\left\|u_{n}-v\right\|^{2} \\
& \leq \beta_{n}\left\|x_{n}-v\right\|^{2}+\left(1-\beta_{n}\right)\left[\left\|x_{n}-v\right\|^{2}-\left\|x_{n}-u_{n}\right\|^{2}\right] \\
& =\left\|x_{n}-v\right\|^{2}-\left(1-\beta_{n}\right)\left\|x_{n}-u_{n}\right\|^{2},
\end{aligned}
$$

and hence

$$
\begin{aligned}
\left\|x_{n+1}-v\right\|^{2} & =\left\|\alpha_{n} f\left(x_{n}\right)+\left(1-\alpha_{n}\right) S y_{n}-v\right\|^{2} \\
& \leq \alpha_{n}\left\|f\left(x_{n}\right)-v\right\|^{2}+\left(1-\alpha_{n}\right)\left\|S y_{n}-v\right\|^{2} \\
& \leq \alpha_{n}\left\|f\left(x_{n}\right)-v\right\|^{2}+\left(1-\alpha_{n}\right)\left\|y_{n}-v\right\|^{2} \\
& \leq \alpha_{n}\left\|f\left(x_{n}\right)-v\right\|^{2}+\left(1-\alpha_{n}\right)\left[\left\|x_{n}-v\right\|^{2}-\left(1-\beta_{n}\right)\left\|x_{n}-u_{n}\right\|^{2}\right] \\
& \leq \alpha_{n}\left\|f\left(x_{n}\right)-v\right\|^{2}+\left\|x_{n}-v\right\|^{2}-\left(1-\alpha_{n}\right)\left(1-\beta_{n}\right)\left\|x_{n}-u_{n}\right\|^{2} .
\end{aligned}
$$

So, we have

$$
\begin{aligned}
\left(1-\alpha_{n}\right)\left(1-\beta_{n}\right)\left\|x_{n}-u_{n}\right\|^{2} & \leq \alpha_{n}\left\|f\left(x_{n}\right)-v\right\|^{2}+\left\|x_{n}-v\right\|^{2}-\left\|x_{n+1}-v\right\|^{2} \\
& \leq \alpha_{n}\left\|f\left(x_{n}\right)-v\right\|^{2}+\left\|x_{n}-x_{n+1}\right\|\left(\left\|x_{n}-v\right\|+\left\|x_{n+1}-v\right\|\right) .
\end{aligned}
$$


Without loss of generality, let us assume that there exists two real numbers $\beta^{*}$ and $\bar{\beta}$ such that $1>\bar{\beta} \geq \beta_{n} \geq \beta^{*}>0$ for all $n \in N$. Hence,

$$
\begin{aligned}
\left(1-\alpha_{n}\right)(1-\bar{\beta})\left\|x_{n}-u_{n}\right\|^{2} & \leq\left(1-\alpha_{n}\right)\left(1-\beta_{n}\right)\left\|x_{n}-u_{n}\right\|^{2} \\
& \leq \alpha_{n}\left\|f\left(x_{n}\right)-v\right\|^{2}+\left\|x_{n}-x_{n+1}\right\|\left(\left\|x_{n}-v\right\|+\left\|x_{n+1}-v\right\|\right) .
\end{aligned}
$$

It follows that $\left\|x_{n}-u_{n}\right\| \rightarrow 0$. We also have

$$
\begin{aligned}
\left\|S u_{n}-x_{n}\right\| & \leq\left\|S y_{n}-x_{n}\right\|+\left\|S u_{n}-S y_{n}\right\| \\
& \leq\left\|S y_{n}-x_{n}\right\|+\left\|u_{n}-y_{n}\right\| \\
& \leq\left\|S y_{n}-x_{n}\right\|+\left\|u_{n}-x_{n}\right\|+\left\|x_{n}-y_{n}\right\| \\
& =\left\|S y_{n}-x_{n}\right\|+\left\|u_{n}-x_{n}\right\|+\left(1-\beta_{n}\right)\left\|x_{n}-S u_{n}\right\| .
\end{aligned}
$$

It follows that

$$
\beta^{*}\left\|S u_{n}-x_{n}\right\| \leq \beta_{n}\left\|S u_{n}-x_{n}\right\| \leq\left\|S y_{n}-x_{n}\right\|+\left\|u_{n}-x_{n}\right\|
$$

Hence, $\left\|S u_{n}-x_{n}\right\| \rightarrow 0$. Since

$$
\left\|S u_{n}-u_{n}\right\| \leq\left\|S u_{n}-x_{n}\right\|+\left\|x_{n}-u_{n}\right\|
$$

we also have $\lim _{n \rightarrow \infty}\left\|S u_{n}-u_{n}\right\|=0$. Next, we show that

$$
\limsup _{n \rightarrow \infty}\left\langle f(z)-z, x_{n}-z\right\rangle \leq 0
$$

where $z=P_{F(S) \cap E P(F)} f(z)$. To show this inequality, we choose a subsequence $\left\{x_{n_{i}}\right\}$ of $\left\{x_{n}\right\}$ such that

$$
\lim _{n \rightarrow \infty}\left\langle f(z)-z, x_{n_{i}}-z\right\rangle=\limsup _{n \rightarrow \infty}\left\langle f(z)-z, x_{n}-z\right\rangle
$$

Since $\left\{u_{n i}\right\}$ is bounded, there exists a subsequence $\left\{u_{n i j}\right\}$ of $\left\{u_{n i}\right\}$ which converges weakly to $w$. Without loss of generality, we can assume that $\left\{u_{n i}\right\} \rightarrow w$. From $\left\|S u_{n}-u_{n}\right\| \rightarrow 0$, we obtain $S u_{n_{i}} \rightarrow w$. Let us show $w \in \operatorname{EP}(F)$. By $u_{n}=T_{r_{n}} x_{n}$, we have

$$
F\left(u_{n}, y\right)+\frac{1}{r_{n}}\left\langle y-u_{n}, u_{n}-x_{n}\right\rangle \geq 0, \quad \forall y \in C
$$

From (A2), we also have

$$
\frac{1}{r_{n}}\left\langle y-u_{n}, u_{n}-x_{n}\right\rangle \geq F\left(y, u_{n}\right)
$$


and hence,

$$
\left\langle y-u_{n_{i}}, \frac{u_{n_{i}}-x_{n_{i}}}{r_{n_{i}}}\right\rangle \geq F\left(y, u_{n_{i}}\right)
$$

Since $\left(u_{n_{i}}-x_{n_{i}}\right) / r_{n_{i}} \rightarrow 0$ and $u_{n_{i}} \rightarrow w$, from (A4), we have

$$
f(y, w) \leq 0, \quad \forall y \in C
$$

For $t$ with $0<t \leq 1$ and $y \in C$, let $y_{t}=t y+(1-t) w$. Since $y \in C$ and $w \in C$, we obtain $y_{t} \in C$ and hence $F\left(y_{t}, w\right) \leq 0$. So we have

$$
0=F\left(y_{t}, y_{t}\right) \leq t F\left(y_{t}, y\right)+(1-t) F\left(y_{t}, w\right) \leq t F\left(y_{t}, y\right)
$$

Dividing by $t$, we get

$$
F\left(y_{t}, y\right) \geq 0
$$

Letting $t \rightarrow 0$ and from (A3), we get

$$
F(w, y) \geq 0
$$

for all $y \in C$ and hence $w \in \operatorname{EP}(F)$. We shall show that $w \in F(S)$. Assume $w \notin F(S)$. Since $u_{n i} \rightarrow w$ and $w \neq S w$, from the Opial theorem [11] we have

$$
\begin{aligned}
\liminf _{i \rightarrow \infty}\left\|u_{n_{i}}-w\right\| & <\liminf _{i \rightarrow \infty}\left\|u_{n_{i}}-S w\right\| \\
& \leq \liminf _{i \rightarrow \infty}\left\{\left\|u_{n_{i}}-S u_{n_{i}}\right\|+\left\|S u_{n_{i}}-S w\right\|\right\} \\
& \leq \liminf _{i \rightarrow \infty}\left\|u_{n_{i}}-w\right\| .
\end{aligned}
$$

This is a contradiction. So, we get $w \in F(S)$. Therefore, $w \in F(S) \cap \operatorname{EP}(F)$. Since $z=$ $P_{F(S) \cap E P(F)} f(z)$, we have

$$
\begin{aligned}
\limsup _{n \rightarrow \infty}\left\langle f(z)-z, x_{n}-z\right\rangle & =\lim _{i \rightarrow \infty}\left\langle f(z)-z, x_{n_{i}}-z\right\rangle \\
& =\lim _{i \rightarrow \infty}\left\langle f(z)-z, u_{n_{i}}-z\right\rangle \\
& =\langle f(z)-z, w-z\rangle \leq 0 .
\end{aligned}
$$


International Journal of Mathematics and Mathematical Sciences

From $x_{n+1}-z=\alpha_{n}\left(f\left(x_{n}\right)-z\right)+\left(1-\alpha_{n}\right)\left(S y_{n}-z\right)$, we have

$$
\begin{aligned}
\left(1-\alpha_{n}\right)^{2}\left\|S y_{n}-z\right\|^{2} & \geq\left\|x_{n+1}-z\right\|^{2}-2 \alpha_{n}\left\langle f\left(x_{n}\right)-z, x_{n+1}-z\right\rangle, \\
\left\|y_{n}-z\right\|^{2} & =\left\|\beta_{n} x_{n}+\left(1-\beta_{n}\right) S u_{n}-z\right\|^{2} \\
& \leq \beta_{n}\left\|x_{n}-z\right\|^{2}+\left(1-\beta_{n}\right)\left\|S u_{n}-z\right\|^{2} \\
& \leq \beta_{n}\left\|x_{n}-z\right\|^{2}+\left(1-\beta_{n}\right)\left\|u_{n}-z\right\|^{2} \\
& \leq\left\|x_{n}-z\right\|^{2} .
\end{aligned}
$$

It follows that

$$
\begin{aligned}
\left\|x_{n+1}-z\right\|^{2} \leq & \left(1-\alpha_{n}\right)^{2}\left\|S y_{n}-z\right\|^{2}+2 \alpha_{n}\left\langle f\left(x_{n}\right)-z, x_{n+1}-z\right\rangle \\
\leq & \left(1-\alpha_{n}\right)^{2}\left\|y_{n}-z\right\|^{2}+2 \alpha_{n}\left\langle f\left(x_{n}\right)-f(z), x_{n+1}-z\right\rangle \\
& +2 \alpha_{n}\left\langle f(z)-z, x_{n+1}-z\right\rangle \\
\leq & \left(1-\alpha_{n}\right)^{2}\left\|x_{n}-z\right\|^{2}+2 \alpha_{n} a\left\|x_{n}-z\right\|\left\|x_{n+1}-z\right\| \\
& +2 \alpha_{n}\left\langle f(z)-z, x_{n+1}-z\right\rangle \\
\leq & \left(1-\alpha_{n}\right)^{2}\left\|x_{n}-z\right\|^{2}+\alpha_{n} a\left\{\left\|x_{n}-z\right\|^{2}+\left\|x_{n+1}-z\right\|^{2}\right\} \\
& +2 \alpha_{n}\left\langle f(z)-z, x_{n+1}-z\right\rangle .
\end{aligned}
$$

Hence

$$
\left\|x_{n+1}-z\right\|^{2} \leq \frac{\left(1-\alpha_{n}\right)^{2}+\alpha_{n} a}{1-\alpha_{n} a}\left\|x_{n}-z\right\|^{2}+\frac{2 \alpha_{n}}{1-\alpha_{n} a}\left\langle f(z)-z, x_{n+1}-z\right\rangle .
$$

From $\alpha_{n} \rightarrow 0$, we know that there exists a positive integer $n_{0}$, such that $1>1-\alpha_{n} a>1 / 2$ for all $n \geq n_{0}$. Then

$$
\begin{aligned}
\frac{\left(1-\alpha_{n}\right)^{2}+\alpha_{n} a}{1-\alpha_{n} a} & =\frac{1-2 \alpha_{n}+\alpha_{n} a}{1-\alpha_{n} a}+\frac{\alpha_{n}^{2}}{1-\alpha_{n} a} \\
& =1-\frac{2(1-a) \alpha_{n}}{1-\alpha_{n} a}+\frac{\alpha_{n}^{2}}{1-\alpha_{n} a} \\
& \leq 1-2(1-a) \alpha_{n}+2 \alpha_{n}^{2}, \quad \forall n \geq n_{0} .
\end{aligned}
$$


Putting above inequality into (3.44), we get

$$
\left\|x_{n+1}-z\right\|^{2} \leq\left(1-2(1-a) \alpha_{n}\right)\left\|x_{n}-z\right\|^{2}+2 \bar{M} \alpha_{n}^{2}+\frac{2 \alpha_{n}}{1-\alpha_{n} a} \sigma_{n}, \quad \forall n \geq n_{0}
$$

where $\bar{M}=\sup \left\{\left\|x_{n}-z\right\|^{2}: n \in N\right\}$, and $\sigma_{n}=\left\langle f(z)-z, x_{n+1}-z\right\rangle$.

It follows from Lemma 2.3 that

$$
x_{n} \longrightarrow z \in F(S) \cap \mathrm{EP}(F) .
$$

It follows from $\left\|x_{n}-u_{n}\right\| \rightarrow 0$ and (3.42) that $u_{n} \rightarrow z$ and $y_{n} \rightarrow z$.

By Theorem 3.1, we can obtain the following new result.

Corollary 3.2. Let $C$ be a nonempty closed convex subset of $H$. Let $S$ be a nonexpansive mapping of $C$ into $H$ such that $F(S) \neq \emptyset$. Let $f$ be a contraction of $H$ into itself and let $\left\{x_{n}\right\}$ and $\left\{y_{n}\right\}$ be sequences generated by $x_{1} \in H$ and

$$
\begin{gathered}
x_{n+1}=\alpha_{n} f\left(x_{n}\right)+\left(1-\alpha_{n}\right) S y_{n}, \\
y_{n}=\beta_{n} x_{n}+\left(1-\beta_{n}\right) S P_{C} x_{n}, \quad \forall n \in N .
\end{gathered}
$$

If $\left\{\alpha_{n}\right\},\left\{\beta_{n}\right\} \subset[0,1]$ satisfy the following conditions:

$$
\begin{array}{cl}
\lim _{n \rightarrow \infty} \alpha_{n}=0, \quad \sum_{n=1}^{\infty} \alpha_{n}=\infty, & \sum_{n=1}^{\infty}\left|\alpha_{n+1}-\alpha_{n}\right|<\infty, \\
0<\liminf _{n \rightarrow \infty} \beta_{n} \leq \limsup _{n \rightarrow \infty} \beta_{n}<1, & \sum_{n=1}^{\infty}\left|\beta_{n+1}-\beta_{n}\right|<\infty,
\end{array}
$$

then, $\left\{x_{n}\right\}$ and $\left\{y_{n}\right\}$ converge strongly to $z \in F(S)$, where $z=P_{F(S)} f(z)$.

Proof. Put $F(x, y)=0$ for all $x, y \in C$ and $r_{n}=1$ for all $n \in N$ in Theorem 3.1. Then, we get $u_{n}=P_{C} x_{n}$. So from Theorem 3.1, the sequences $\left\{x_{n}\right\}$ and $\left\{y_{n}\right\}$ converge strongly to $z \in F(S)$, where $z=P_{F(S)} f(z)$.

Remark 3.3. Theorem 3.1 and Corollary 3.2, respectively, extend and generalize Theorem 3.2 and Corollary 3.3 in [7] from the Mann iterative form to the Ishikawa iterative form.

\section{Acknowledgments}

The authors would like to express their thanks to the referees for helpful suggestions. This research was supported by the National Natural Science Foundation of China (Grants 10771228 and 10831009), the Natural Science Foundation of Chongqing (Grant no. CSTC, 2009BB8240), and the Research Project of Chongqing Normal University (Grant 08XLZ05). 


\section{References}

[1] S. D. Flåm and A. S. Antipin, "Equilibrium programming using proximal-like algorithms," Mathematical Programming, vol. 78, no. 1, pp. 29-41, 1997.

[2] E. Blum and W. Oettli, "From optimization and variational inequalities to equilibrium problems," The Mathematics Student, vol. 63, no. 1-4, pp. 123-145, 1994.

[3] K. Goebel and W. A. Kirk, Topics in Metric Fixed Point Theory, vol. 28 of Cambridge Studies in Advanced Mathematics, Cambridge University Press, Cambridge, UK, 1990.

[4] A. Moudafi, "Viscosity approximation methods for fixed-points problems," Journal of Mathematical Analysis and Applications, vol. 241, no. 1, pp. 46-55, 2000.

[5] H.-K. Xu, "Viscosity approximation methods for nonexpansive mappings," Journal of Mathematical Analysis and Applications, vol. 298, no. 1, pp. 279-291, 2004.

[6] P. L. Combettes and S. A. Hirstoaga, "Equilibrium programming in Hilbert spaces," Journal of Nonlinear and Convex Analysis, vol. 6, no. 1, pp. 117-136, 2005.

[7] S. Takahashi and W. Takahashi, "Viscosity approximation methods for equilibrium problems and fixed point problems in Hilbert spaces," Journal of Mathematical Analysis and Applications, vol. 331, no. 1, pp. 506-515, 2007.

[8] S. Ishikawa, "Fixed points by a new iteration method," Proceedings of the American Mathematical Society, vol. 44, pp. 147-150, 1974.

[9] W. Takahashi, Introduction to Nonlinear and Convex Analysis, Yokohama Publishers, Yokohama, Japan, 2009.

[10] H. K. Xu, "An iterative approach to quadratic optimization," Journal of Optimization Theory and Applications, vol. 116, no. 3, pp. 659-678, 2003.

[11] Z. Opial, "Weak convergence of the sequence of successive approximations for nonexpansive mappings," Bulletin of the American Mathematical Society, vol. 73, pp. 591-597, 1967. 


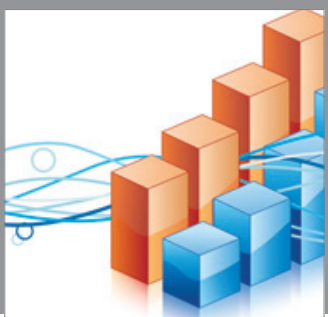

Advances in

Operations Research

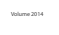

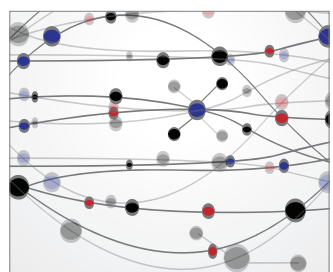

\section{The Scientific} World Journal
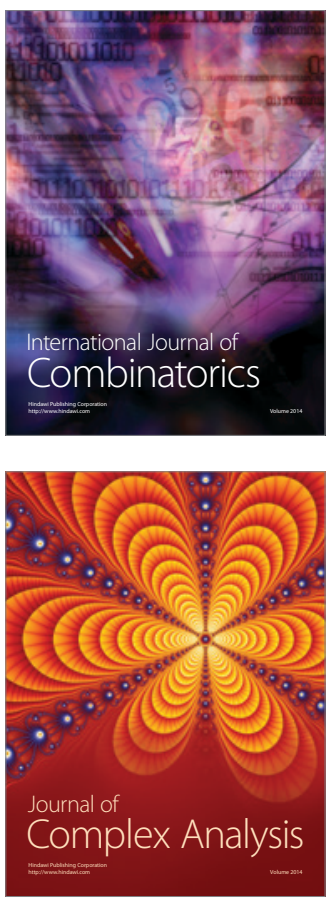

International Journal of

Mathematics and

Mathematical

Sciences
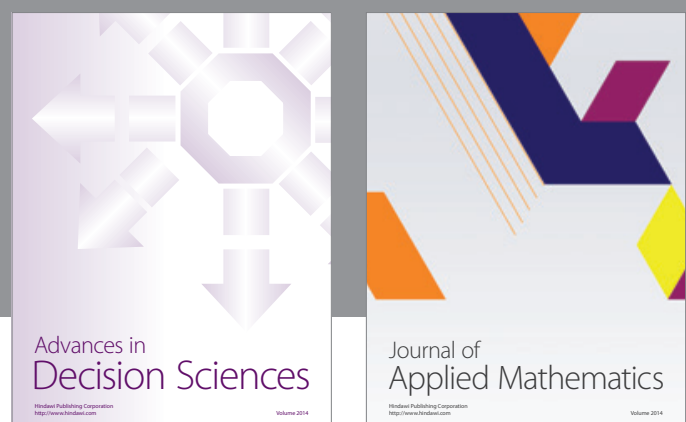

Journal of

Applied Mathematics
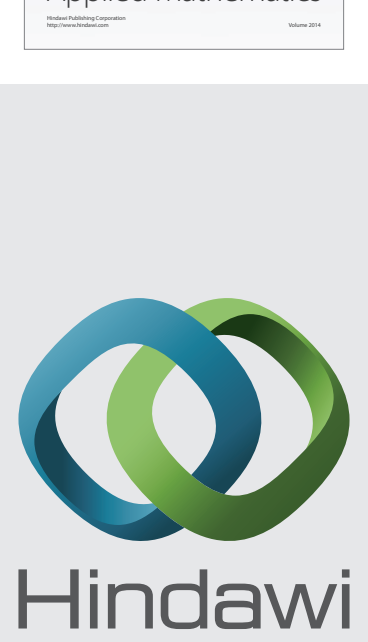

Submit your manuscripts at http://www.hindawi.com
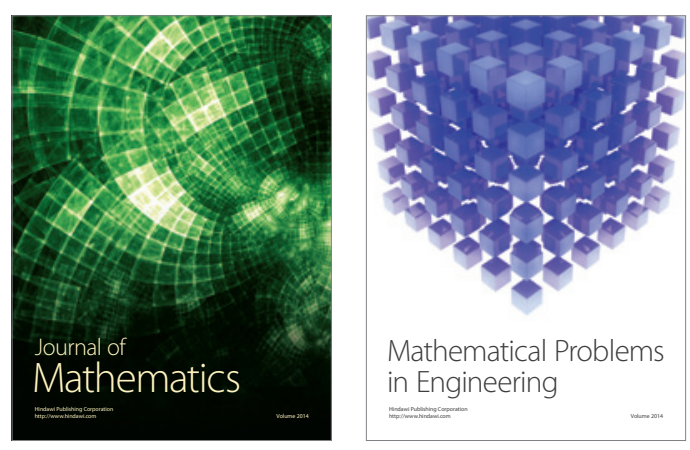

Mathematical Problems in Engineering
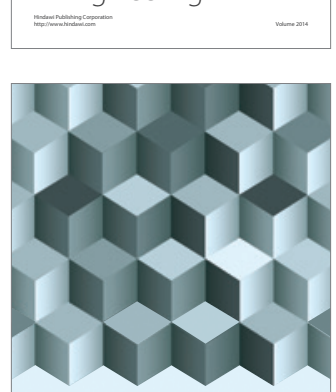

Journal of

Function Spaces
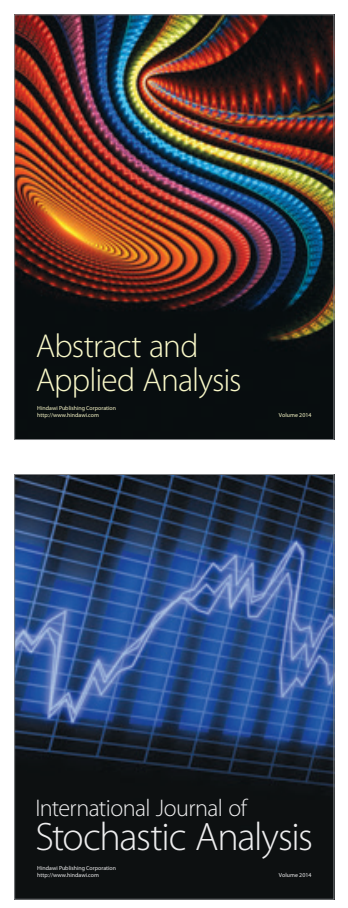

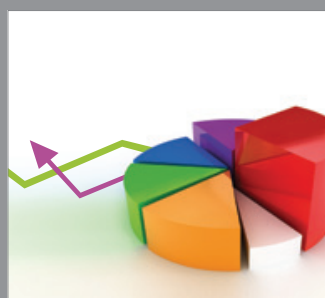

ournal of

Probability and Statistics

Promensencen
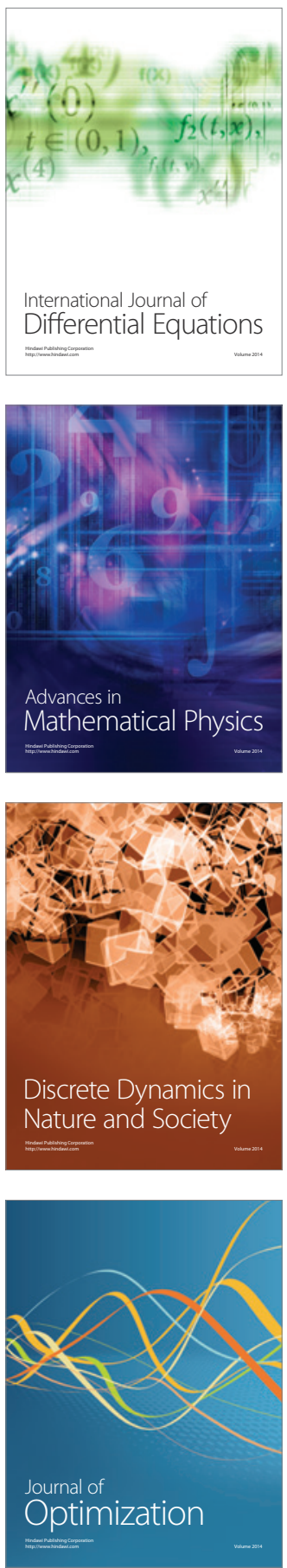